\begin{tabular}{|c|c|}
\hline & $\begin{array}{l}\text { International Journal of Trend in Scientific } \\
\text { Research and Development (IJTSRD) }\end{array}$ \\
\hline 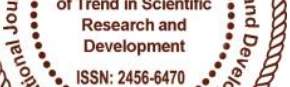 & International Open Access Journal \\
\hline 00 & ISSN No: 2456 - 6470 | www.ijtsrd.com | Volume - 2 | Issue -3 \\
\hline
\end{tabular}

\title{
Spot Speed Study for Accident Analysis and Mitigation Measures on Wardha-Pawnar Road
}

\author{
S. V. Nimkar \\ IV $^{\text {th }}$ Sem, M.E Civil (TE\&M) \\ DRGITR, Amravati, \\ Maharashtra, India
}

\author{
Dr. M. R. Vyawhare \\ Ph.D.M.Tech (Structure), \\ HOD Civil Department \\ DRGITR, Amravati, \\ Maharashtra, India
}

\author{
Prof. V. S. Umap \\ M. Tech (Structures), \\ DRGITR, Amravati, \\ Maharashtra, India
}

\begin{abstract}
Accident is a term used to represent sudden fatal event with multiple reasons, persons, properties and things evolved. The main cause of accidents by many researches is concluded as speed factor \& speed is the parameter which mainly depends upon the owner of vehicle and class of road. This parameter is divided in to spot speed \& journey speed. The paper contains the full part of spot speed which was studied and resulted on project location and obtained results are further considered in analysis and mitigation measures.
\end{abstract}

\section{INTRODUCTION}

Spot sped-It is the instantaneous speed of a vehicle at a specified location. Spot speed study is the one key factor which can give an idea about the accidents causes hence for evaluation and remedial measure of accident $\mathrm{s}$ this speed study has been carried out the method used for study is long base direct timing procedure on a patch of Wardha Pawnar road. The results from the study are presented in tabular form for analyzing variations in speed and correlate the permissible maximum speed for moving vehicles on selected road patch and then suggestion will be suggested.

\section{STUDY AREA}

The existing location for spot speed study is on Wardha Pawnar road of $3.4 \mathrm{~km}$ patch started from hotel delight to end at Dham river bridge. The Wardha Pawnar road is the patch of Aurangabad-Nagpur road designated as NH-361
It is a two way single lane road with width at carriage way as $6.90 \mathrm{~m}$ and $1.80 \mathrm{~m}$ shoulders on both sides. The pavement is a high type bituminous type.

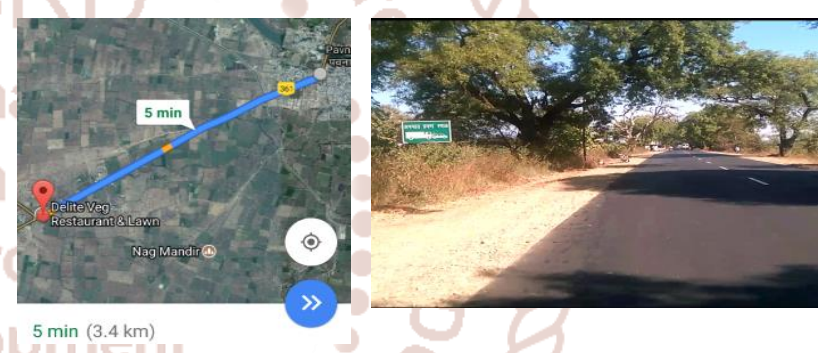

\section{METHODOLOGY}

Following are the methods of spot speed study

\section{Long base}

a) Radar speedometer

b) Photographic method

2. Short Base

a) Direct timing

b) Enoscope

c) Pressure and contact tube

Here we used long base direct timing procedure This method consists of taking two reference points on pavement at a suitable distance apart can be taken from the following table: 
International Journal of Trend in Scientific Research and Development (IJTSRD) ISSN: 2456-6470

\begin{tabular}{|l|l|}
\hline $\begin{array}{l}\text { STREAM SPEED IN } \\
\text { KMPH }\end{array}$ & $\begin{array}{l}\text { LENGTH } \\
\text { METERS }\end{array}$ \\
\hline Less than 40 & 27 \\
\hline $40-65$ & 54 \\
\hline More than 65 & 81 \\
\hline
\end{tabular}

The observer starts and stops stop watch as a vehicle crosses these two marks. From the known distance and measured time intervals speeds are calculated.

The modifications in above process is that the two observers are taking reading in such a way that one observer stand at 1 st point and second observer stands at 2 nd reference point. When vehicle passes signal the second observer stars watch and when vehicle cross second observer he stops watch in such a way timings of various vehicles under consideration are recorded and used for further calculations.

\section{OBSERVATIONS}

The observations are done at three patches of road.

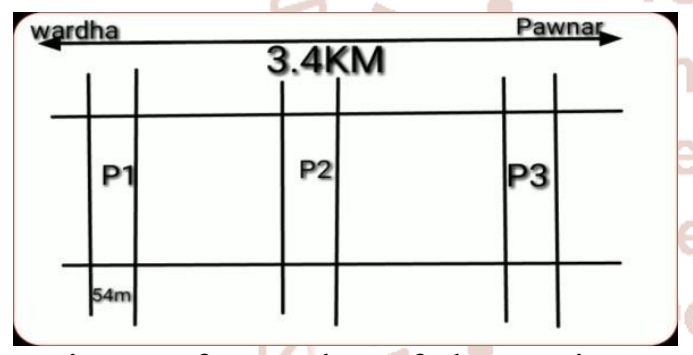

Diagram for patches of observations.

Observations for patch no. 1 are as follows

Location: - Near the bridge of Dham river on Wardha Pawnar road.

Time-10.00a.m

Date:-28/01/2018

Patch length:-54 m.

SR. TIME FOR PASSING 54M PATCH

NO LENGTH FOR DIFFERENT CATEGORY

\begin{tabular}{|l|l|l|l|}
\multirow{2}{*}{} & \multicolumn{3}{|l}{ OF VEHICLES IN SECONDS } \\
\cline { 2 - 4 } & 2WHEELER & 3WHEELER & 4WHEELER \\
\hline 1$)$ & 5.30 & 3.61 & 7.43 \\
\hline 2$)$ & 3.64 & 3.55 & 6.41 \\
\hline 3$)$ & 2.99 & 3.94 & 6.53 \\
\hline 4$)$ & 2.99 & 4.87 & 6.07 \\
\hline 5$)$ & 3.94 & 5.77 & 5.77 \\
\hline 6$)$ & 3.88 & 5.34 & 5.65 \\
\hline 7$)$ & 4.35 & 5.33 & 6.01 \\
\hline 8$)$ & 4.65 & 5.89 & 4.32 \\
\hline 9$)$ & 5.13 & 6.02 & 4.39 \\
\hline 10$)$ & 4.88 & 4.36 & 4.91 \\
\hline
\end{tabular}

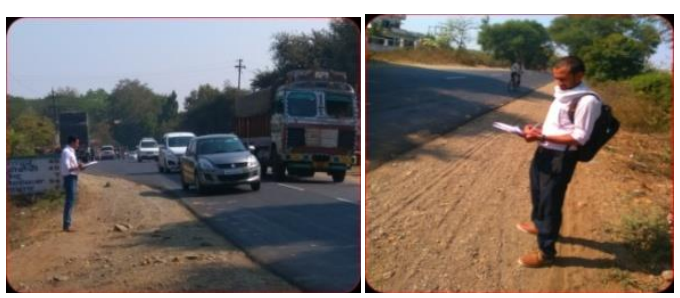

Photographs taken for patch no.1

Observations for patch no. 2 are as follows.

Location: Near mama bhang dargah on Wardha Pawnar road.

Time-2.p.m Date:-28/01/2018 Patch length:-54 m.

SR.NO TIME FOR PASSING 54M PATCH LENGTH FOR DIFFERENT CATEGORY OF VEHICLES IN SECONDS

\begin{tabular}{|l|l|l|l|}
\hline \multirow{2}{*}{} & 2WHEELER & 3 WHEELER & 4WHEELER \\
\hline 1$)$ & 4.37 & 6.02 & 3.66 \\
\hline 2$)$ & 4.44 & 6.37 & 3.32 \\
\hline 3$)$ & 3.94 & 4.47 & 4.06 \\
\hline 4$)$ & 4.01 & 6.01 & 4.14 \\
\hline 5$)$ & 4.35 & 5.89 & 4.32 \\
\hline 6$)$ & 5.55 & 5.33 & 3.00 \\
\hline 7$)$ & 5.71 & 6.41 & 4.46 \\
\hline 8$)$ & 4.82 & 3.53 & 3.91 \\
\hline 9$)$ & 4.08 & 5.80 & 3.18 \\
\hline 10$)$ & 3.37 & 6.07 & 5.22 \\
\hline
\end{tabular}

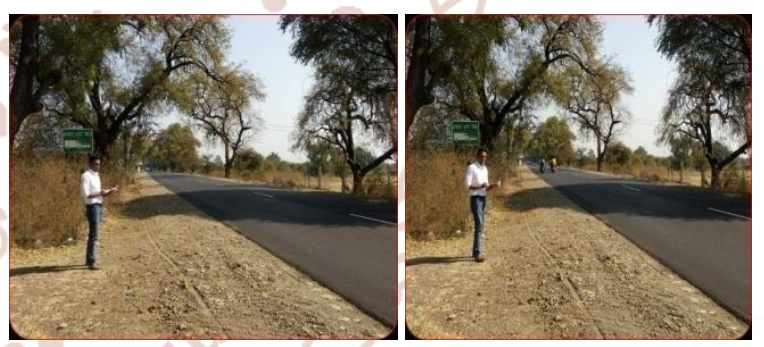

Photographs taken for patch no. 2

Observations for patch no. 3 are as follows.

Location:-Near hotel Delight, Wardha Pawnar road.

Time:-5.00 p.m. Date-28/01/2018 Patch length:-54m

SR. TIME FOR PASSING 54m PATCH LENGTH

NO FOR DIFFERENT CATEOGARY OF VEHICLES IN SECOND

\begin{tabular}{l|l|l|} 
2WEELER & 3WHEELER & 4WHEELER
\end{tabular}

\begin{tabular}{|l|l|l|l|}
\hline 1$)$ & 3.30 & 3.88 & 4.27 \\
\hline 2$)$ & 3.41 & 4.76 & 4.44
\end{tabular}

\begin{tabular}{|l|l|l|l|}
\hline 2) & 3.41 & 4.76 & 4.44 \\
\hline 3$)$ & 3.02 & 3.82 & 4.52
\end{tabular}

\begin{tabular}{l|l|l|l|}
\hline 3$)$ & 3.02 & 3.82 & 4.52 \\
\hline 4$)$ & 3.20 & 3.98 & 3.34 \\
\hline
\end{tabular}

\begin{tabular}{|l|l|l|l}
\hline 4$)$ & 3.20 & 3.98 & 3.34 \\
\hline 5$)$ & 2.67 & 4.56 & 3.65 \\
\hline 6$)$ & 3.89 & 4.999
\end{tabular}

\begin{tabular}{|l|l|l|l|}
\hline 6$)$ & 3.89 & 4.99 & 3.79 \\
\hline 7$)$ & 4.07 & 4.04 & 4.66 \\
\hline 8$)$ & 4.44 & 4.55 & 3.82 \\
\hline 9$)$ & 3.71 & 4.93 & 4.18 \\
\hline 10$)$ & 3.66 & 5.03 & 3.01 \\
\hline
\end{tabular}


International Journal of Trend in Scientific Research and Development (IJTSRD) ISSN: 2456-6470

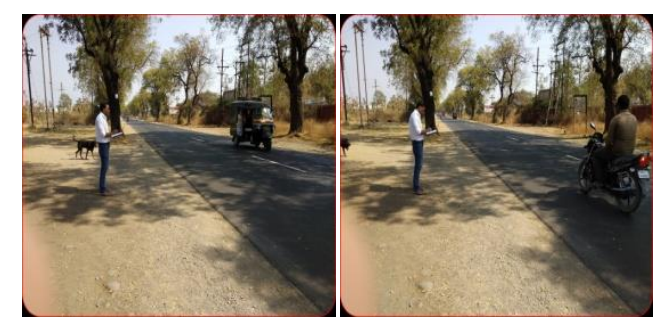

Photograph taken for patch no.3

\section{CALCULATIONS}

Calculations are done in following steps

1) To obtain speed in kmph use following formula.

$$
\mathrm{V}=(\mathrm{D} / \mathrm{T})^{*}(18 / 5)
$$

2) Divide the speed in speed groups.

3) Calculate $\%$ frequency

4) Calculate cumulative $\%$ frequency

5) Draw a graph mid speed vs. cumulative \% frequency.

6) Find the $85^{\text {th }}, 98$ th and $15^{\text {th }} \%$ speed.

Repeat above procedure for each patch

For patch no.1

\begin{tabular}{|l|l|l|l|l|}
\hline $\begin{array}{l}\text { Speed } \\
(\mathrm{Kmph})\end{array}$ & $\begin{array}{l}\text { Mid } \\
\text { Speed }\end{array}$ & $\begin{array}{l}\text { Frequ } \\
\text { ency }\end{array}$ & $\begin{array}{l}\% \\
\text { Frequenc } \\
\mathrm{y}\end{array}$ & $\begin{array}{l}\text { Cumulative } \\
\% \text { ernatio } \\
\text { Frequency }\end{array}$ \\
\hline $0-10$ & 5 & 0 & 0 & 0 \\
\hline $10-20$ & 15 & 0 & 0 & 0 \\
\hline $20-30$ & 25 & 3 & 10 & 10 Reseave \\
\hline $30-40$ & 35 & 13 & 43.3 & 53.3 \\
\hline $40-50$ & 45 & 9 & 30 & 83.3 \\
\hline $50-60$ & 55 & 3 & 10 & 93.3 \\
\hline $60-70$ & 65 & 2 & 6.6 & 99.9 \\
\hline
\end{tabular}

For patch no.2

\begin{tabular}{|c|c|c|c|c|}
\hline $\begin{array}{c}\text { Speed } \\
\text { (Kmph) }\end{array}$ & $\begin{array}{c}\text { Mid } \\
\text { Speed }\end{array}$ & $\begin{array}{c}\text { Freq } \\
\text { uenc } \\
\mathrm{y}\end{array}$ & $\begin{array}{c}\% \\
\text { Frequency }\end{array}$ & $\begin{array}{c}\text { Cumulative } \\
\% \\
\text { Frequency }\end{array}$ \\
\hline $0-10$ & 5 & 0 & 0 & 0 \\
\hline $10-20$ & 15 & 0 & 0 & 0 \\
\hline $20-30$ & 25 & 0 & 0 & 0 \\
\hline $30-40$ & 35 & 12 & 40 & 40 \\
\hline $40-50$ & 45 & 12 & 40 & 80 \\
\hline $50-60$ & 55 & 5 & 16.6 & 96.6 \\
\hline $60-70$ & 65 & 1 & 3.3 & 99.9 \\
\hline
\end{tabular}

For patch no.3

\begin{tabular}{|c|c|c|c|c|}
\hline $\begin{array}{c}\text { Speed } \\
(\text { Kmph })\end{array}$ & $\begin{array}{c}\text { Mid } \\
\text { Speed }\end{array}$ & $\begin{array}{c}\text { Frequ } \\
\text { ency }\end{array}$ & $\begin{array}{c}\% \\
\text { Frequen } \\
\text { Cy }\end{array}$ & $\begin{array}{c}\text { Cumulativ } \\
\text { E\% } \\
\text { Frequency }\end{array}$ \\
\hline $0-10$ & 5 & 0 & 0 & 0 \\
\hline $10-20$ & 15 & 0 & 0 & 0 \\
\hline $20-30$ & 25 & 0 & 0 & 0 \\
\hline $30-40$ & 35 & 3 & 10 & 10 \\
\hline $40-50$ & 45 & 14 & 46.6 & 56.6 \\
\hline $50-60$ & 55 & 10 & 33.3 & 89.9 \\
\hline $60-70$ & 65 & 3 & 10 & 99.9 \\
\hline
\end{tabular}

By drawing graph mid speed vs. cumulative \% frequency, we got following results

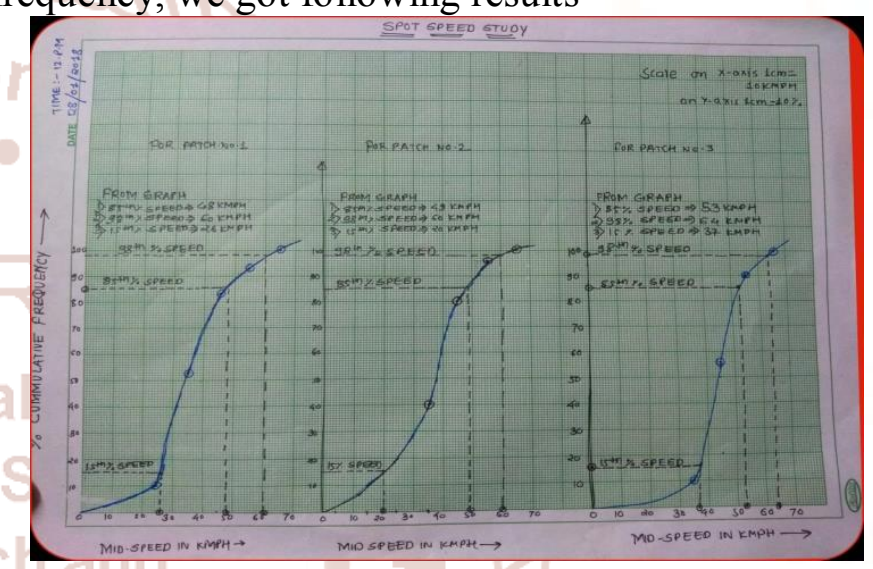

From graph following results are obtained.

For patch no.1

1.85 th $\%$ mid speed $=48 \mathrm{kmph}$

$2.98 \mathrm{th} \%$ mid speed $=60 \mathrm{kmph}$

3.15 th $\%$ mid speed $=26 \mathrm{kmph}$

For patch no. 2

$1.85 \mathrm{th} \%$ midspeed $=49 \mathrm{kmph}$

$2.98 \mathrm{th} \%$ midspeed $=60 \mathrm{kmph}$

$3.15 \mathrm{th} \%$ midspeed $=20 \mathrm{kmph}$

For patch no.3

$1.85^{\text {th }} \%$ mid speed $=53 \mathrm{kmph}$ $2.98^{\text {th }} \% \mathrm{mid}$ speed $=64 \mathrm{kmph}$

$3.15^{\text {th }} \%$ mid speed $=37 \mathrm{kmph}$

Average $85^{\text {th }} \%$ speed $=50 \mathrm{kmph}$

Average $98^{\text {th }} \%$ speed $=61.33 \mathrm{kmph}$

Average $15^{\text {th }} \%$ speed $=27.66 \mathrm{kmph}$ 


\section{CONCLUSION}

From the above results it is seen than the average speed of vehicles is $61.33 \mathrm{kmph}$ For $98^{\text {th }} \%$ which is also taken for designing of road geometries hence for speed of $61.33 \mathrm{kmph}$ road geometries are of sufficient capacities therefore for the selected location speed is not main factor for accidents.

\section{REFERENCES}

1. Khanna \& Justo, Highway engineering and geometric design Nem Chand \& Bros.2011

2. Kadiyali L.R, Traffic engineering and transport planning, khanna publishers new Delhi.2009

3. Pocket book for highway engineers, Indian road congress, Delhi

4. A.K.M Abir \& Md Sami Hasnaine, Traffic speed study.

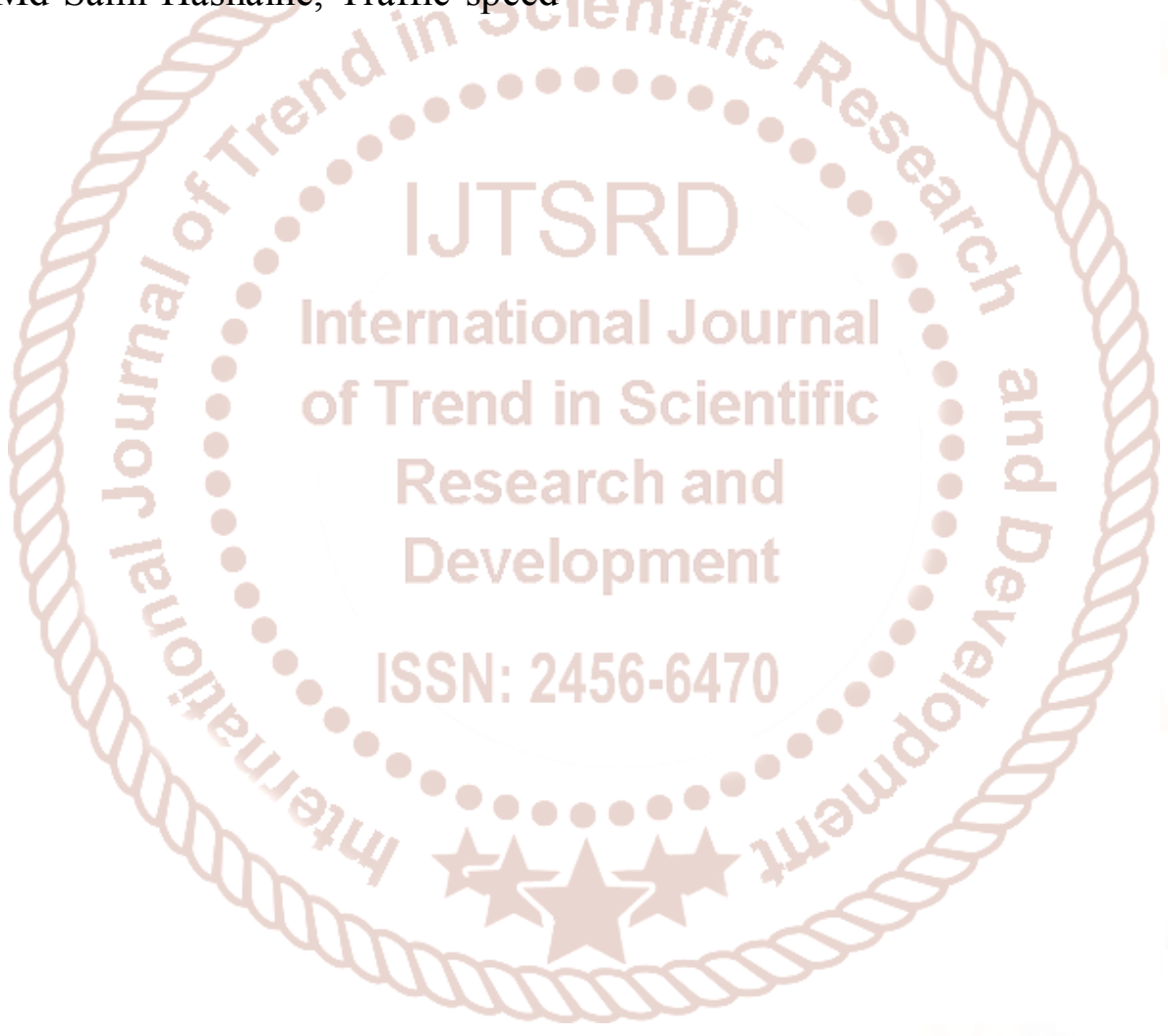

